\title{
Receiver Design Principles for Estimation over Fading Channels
}

\author{
Yasamin Mostofi and Richard M. Murray \\ California Institute of Technology \\ Pasadena, CA 91125, USA
}

\begin{abstract}
In this paper we consider estimation of dynamical systems over wireless communication channels. We show that the communication protocols suitable for non real-time applications like data networks may not be entirely applicable for estimation and control of a rapidly changing dynamical system. We then develop new design paradigms for these applications to show how noisy packets should be handled in the receiver. We prove that, in the presence of a crosslayer feedback, keeping all the packets will both maximize the stability range and minimize the estimation error variance. In the absence of such a feedback, we show that keeping all the packets still maximizes the stability range, independent of the shape of the communication noise profile. In order to optimize the performance, in the absence of a cross-layer feedback, we prove that packet drop should be designed to balance information loss and communication noise.
\end{abstract}

\section{INTRODUCTION}

There has recently been considerable interest in estimation and control over wireless links. Advances in technology have resulted in an abundance of cheap embedded units equipped with sensing, processing, communication and actuation capabilities. This has resulted in a wide range of sensor/actuator network applications [1]. Communication plays a key role in the overall performance of such networks as both sensor measurements and control commands are transmitted over wireless links.

In this paper we consider a mobile sensor that is observing a dynamical system. It transmits its observation over a wireless link to a remote node that is in charge of estimation using a Kalman filter. This is a fundamental problem that can arise in networked sensing, estimation and control. Considering the impact of communication links on estimation and control is an emerging area of research. At this point, it is not yet clear what the right communication design strategies are for such delay-sensitive applications. Most of the current work has applied design principles suitable for non real-time applications like data networks when considering estimation and control over wireless links. Data networks are not as sensitive to delays since the application is not real time. The receiver, therefore, can afford to drop erroneous packets and wait for retransmission. Control applications, on the other hand, are typically delay sensitive as we are racing against the dynamics of the system. Therefore, applying the same design strategies of data networks may not be appropriate.

Micheli et al. investigated impact of packet loss on estimation by considering random sampling of a dynamical system [2]. This is followed by the work of Sinopoli et al. which derived a range for the maximum tolerable probability of packet loss to maintain stability [3]. Liu et al. extended that work to the case of two sensors [4]. These works applied data network design principles, better suited for non real-time applications. They assumed a receiver that drops those packets that contain any amount of error and waits to receive packets that are noise-free. This can result in loss of performance and instability as we are racing against dynamics of the system.

We took a different approach in [5] and proved that keeping all the packets and utilizing a cross-layer feedback can prevent the instability that is introduced when erroneous packets are dropped. In this paper we extend that work to establish fundamentals of design strategies for delaysensitive estimation applications. We consider both cases that the receiver is equipped with a cross-layer feedback as well as scenarios that such a feedback is not supported by the receiver. Instead of applying data network design principles, we ask what the right design framework is for such applications. The main question this paper addresses is the following: "How should noisy packets be handled in the receiver?". We answer this question both in terms of stability and performance (minimizing the estimation error variance). Furthermore, we explore the role of a cross-layer feedback and its impact on the optimum design. We prove that stability condition stays the same independent of the availability of a cross-layer feedback or the shape of the communication noise profile. To optimize the performance, when a cross-layer feedback is available, we show that the receiver should keep all the packets. In the absence of such a feedback, we prove that packet drop should be designed to balance information loss and communication noise in order to optimize the performance.

\section{SYSTEM MODEL}

Consider a mobile sensor observing a linear dynamical system as follows:

$$
\begin{gathered}
x[k+1]=A x[k]+w[k] \\
y[k]=C x[k]+v[k],
\end{gathered}
$$

where $x[k]$ and $y[k]$ represent the state and observation respectively. $w[k]$ and $v[k]$ represent zero-mean process and observation noises with variances of $Q$ and $R$ respectively. In this paper, we assume scalar quantities to facilitate 
mathematical derivations. We are interested in estimating unstable dynamics and therefore we consider cases where $|A|>1$. The sensor then transmits its observation over a wireless fading channel to a remote node, which is in charge of estimation. Since estimation of dynamical systems over time-varying links has not been studied extensively, we keep our analysis general by considering time-varying channels. Therefore, our terminology is geared towards wireless fading channels. However, the analysis and results are applicable to any fixed or time-varying communication link such as wireless, wireline, underwater, ....

\section{A. Physical Layer: Wireless Communication}

In this part we will see how to model the impact of a noisy communication channel on the observation. The sensor quantizes the observation, $y[k]$, transforms it into a packet of bits and transmits it over a fading channel. The remote node will receive a noisy version of the transmitted data. Let $\hat{y}[k]$ represent output of the receiver at the remote node. Then,

$$
\hat{y}[k]=y[k]+n[k] .
$$

It should be noted that $\hat{y}[k]$ represents output of the physical layer, not its input, at the remote node. $n[k]$ represents zero-mean communication noise at time $k$ and does not necessarily have a Gaussian distribution. Let $\sigma_{n}^{2}[k]$ represent the variance of $n[k]$ at $k^{t h}$ transmission:

$$
\sigma_{n}^{2}[k]=E\left(n^{2}[k]\right) .
$$

A fundamental parameter that impacts the performance of a communication channel is the received Signal to Noise Ratio [6]. As the sensor moves, the remote node will experience different received Signal to Noise Ratios. Let $\Upsilon[k]$ represent the instantaneous received Signal to Noise Ratio at $k^{t h}$ transmission. Then $\sigma_{n}^{2}[k]$ will be a function of $\Upsilon[k]$ :

$$
\sigma_{n}^{2}[k]=F(\Upsilon[k]),
$$

where function $F$ is a decreasing function that depends on the transmitter/receiver designs such as quantization, modulation and coding as well as the transmission environment.

Depending on the receiver design, there can be a packet drop mechanism deployed in the receiver. Let $P_{d r o p}[k]$ represent the probability that the receiver drops the $k^{\text {th }}$ packet. $P_{d r o p}[k]$ can be presented as a function of $\Upsilon[k]$ as well:

$$
P_{d r o p}(k)=G(\Upsilon[k]),
$$

where $G$ is a non-increasing function. Fig. 1 shows a sample $P_{\text {drop }}$ as a function of $\Upsilon$ (solid line). It should be noted that the receiver may not decide on dropping packets directly based on $\Upsilon[k]$. Since any other utilized measure is a function of $\Upsilon[k]$, we find it useful to express $P_{d r o p}$ as a function of this fundamental parameter. $G$ is also a function of receiver and transmitter technologies. Functions $F$ and $G$ provide the abstraction necessary to consider the impact of physical layer in the higher application layer.
$\Upsilon[k]$ is a stationary stochastic process with its distribution a function of the environment and level of the mobility of the sensor. We take $\Upsilon[k]$ to be independent from one transmission to the next. This will be the case as long as the time interval between consecutive transmissions is bigger than channel coherence time [7]. In this paper, we do not make any assumption on the distribution of $\Upsilon$. Only when we want to provide an example, we take $\Upsilon$ to be exponentially distributed ${ }^{1}$. To ease mathematical derivations we will approximate function $G$ with the following:

$$
P_{\text {drop }}(\Upsilon[k])=\left\{\begin{array}{cc}
0 & \Upsilon[k] \geq \Upsilon_{T} \\
1 & \text { else }
\end{array}\right.
$$

This means that the receiver keeps those packets with received Signal to Noise Ratio above a designated threshold: $\Upsilon_{T}$. This approximation is shown in Fig. 1 (start line).

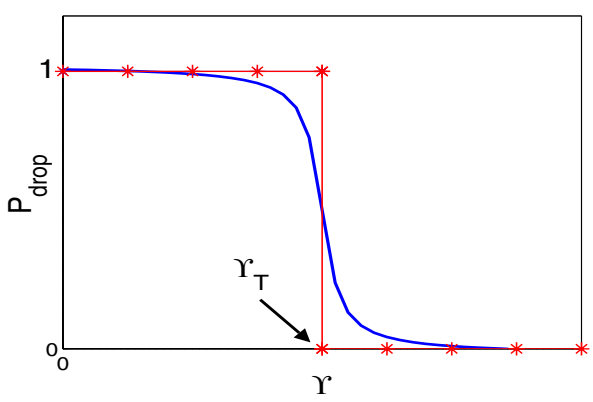

Fig. 1. Solid line: an example of packet loss probability as a function of Signal to Noise Ratio, star line: rectangular approximation

\section{B. Application Layer: Estimation}

The remote node estimates the state based on the received observation using a Kalman filter [8]. Let $\hat{x}[k]$ represent the estimate of $x[k]$ given $\hat{y}[0], \ldots, \hat{y}[k-1]$ at the remote node. Then $e[k]$ represents the corresponding estimation error variance given $\Upsilon[k-1], \ldots, \Upsilon[0]$ :

$$
e[k]=E\left\{(x[k]-\hat{x}[k])^{2}\right\} \Upsilon[k-1], \ldots, \Upsilon[0] \cdot
$$

This is different from typical Kalman filtering since $e[k]$ is a function of channel statistics through $\Upsilon[k-1], \ldots, \Upsilon[0]$. To obtain $E(e[k]), e[k]$ should be averaged over the joint distribution of $\Upsilon[k-1], \ldots, \Upsilon[0]$. There will be different forms of recursion for $e[k]$ depending on the availability of a cross-layer feedback in the receiver.

\section{Cross-Layer Feedback}

In networked sensing, estimation and control, application layer will be in charge of estimation and control whereas the knowledge of the quality of the communication link will be available in the physical layer. A cross-layer feedback, in this case, refers to a feedback from physical layer to the application layer that carries some information on the

\footnotetext{
${ }^{1}$ Taking Signal to Noise Ratio to be exponentially distributed is a common model for outdoor fading channels with no line-of-sight [7].
} 
quality of the link such as communication noise variance or channel Signal to Noise Ratio. While presence of such a feedback can play a key role in the overall performance and the optimum design typically involves one, the receiver architecture may not support it. Therefore, in this paper we will consider scenarios where such a feedback is available at the receiver as well as cases where it is not supported by the architecture.

\section{Scenario\# 1: IdEAL NoISE Profile}

When considering estimation and control over wireless links, the current work in the literature considers estimation over fixed wireless channels. Furthermore, it applies data network design principles by assuming a receiver that drops those packets that contain any amount of error. The packets that are kept in the receiver, therefore, are assumed to be noise-free. We refer to this assumption on the communication noise profile as "ideal noise profile" throughout the paper. Furthermore, we refer to this design strategy, which applies data network protocols, as "scenario\#1". Such an assumption translates to the following recursion for the estimation error variance [3]:

$$
e[k+1]=A^{2} e[k]-\frac{A^{2} e^{2}[k] C^{2}}{R+C^{2} e[k]+S_{1}(\Upsilon[k])}+Q,
$$

where $\quad S_{1}[k] \quad=\quad\left\{\begin{array}{cc}0 & \Upsilon[k] \geq \Upsilon_{T} \\ \infty & \text { else }\end{array}\right.$
$E\left(P_{\text {drop,scenario\#1 })}<A^{-2}\right.$ is required for stability. and

\section{RECEIVER DESIGN OPTIMIZATION}

For non real-time applications like data networks, the receiver can afford to drop erroneous packets and wait for retransmission. Considering packets to be noise-free once they are kept in the receiver, therefore, is a reasonable model for these applications. However, estimation of a rapidly changing dynamical system is delay sensitive. Dropping erroneous packets can result in loss of information, can reduce the useful transmission rate and can lead to instability. Therefore, the receiver can not afford to wait for receiving noise-free packets. The main issue is then to find the right strategy for handling the received, possibly noisy, packets. The optimum receiver design can also change depending on the availability of a cross-layer feedback, which should be taken into account in the analysis.

We have shown in [5] that keeping all the packets and utilizing a cross-layer feedback at the receiver can prevent the instability that is introduced when erroneous packets are dropped. The analysis of [5] was provided for one class of communication noise profiles and an exponentially distributed Signal to noise Ratio. Furthermore, not all the receivers can support a cross-layer feedback and the optimum receiver design, in the absence of such a feedback, is yet to be determined.

This Section will derive receiver design theories for delay-sensitive estimation over wireless links without making any assumption on channel characteristics. We will

\begin{tabular}{|c|c|c|c|}
\hline & \multicolumn{2}{|c|}{ NON-IDEAL } & IDEAL \\
& NOISE PROFILE & NOISE \\
& Cross-Layer & No Cross-Layer & PRILE \\
\cline { 2 - 3 } & $\begin{array}{c}\text { Scenario\#3 } \\
\text { PACKET }\end{array}$ & $\begin{array}{c}\text { Scenario\#2 } \\
*\end{array}$ & $\begin{array}{c}\text { Scenario\#1 } \\
\text { Studied by } \\
\text { Sinopoli et al. }\end{array}$ \\
\hline KEEP & $*$ & $*$ & Not Possible \\
ALL & $\begin{array}{c}\text { Studied by } \\
\text { Mostofi et al. } \\
\end{array}$ & & \\
\hline
\end{tabular}

TABLE I

DIFFERENT RECEIVER DESIGNS- THE ASTERISKS INDICATE CASES NOT PREVIOUSLY CONSIDERED WHICH THIS PAPER ADDRESSES

analyze the performance and find the stability conditions for the following cases:

1) The receiver cannot keep all the packets and cannot provide a cross-layer feedback,

2) The receiver cannot keep all the packets but can provide a cross-layer feedback for those packets kept,

3) The receiver can keep all the packets but cannot provide a cross-layer feedback,

4) Finally, the receiver can keep all the packets and is equipped with a cross-layer feedback.

Considering the aforementioned cases will provide insights into receiver design strategies. Table I summarizes different possible scenarios. The first row, "PACKET DROP", refers to the cases where the receiver deploys a type of packet drop mechanism. The second row, "KEEP ALL", refers to the cases where the receiver is keeping all the packets. "IDEAL NOISE PROFILE" refers to the assumption that any packet that is kept in the receiver is noise-free. Scenario\#1 denotes cases that apply data network design principles, as discussed in Section III. If all the packets are kept in the receiver, then packets can not be considered noise-free. This scenario is therefore crossed out in Table I.

When considering non-ideal noise profiles, there will be four possibilities as marked in Table I. In both scenario\#2 and 3 , the receiver is equipped with a form of packet drop mechanism. In scenario\#3 a cross-layer feedback is available for those packets that are kept in the receiver. The second row of Table I, case of keeping all the packets, can be considered as a special case of the first row with probability of packet drop set to zero. The goal of this section is to study different scenarios of Table I for a general communication noise variance and channel distribution. The asterisks indicate the scenarios that have not been considered before. We will study scenario\#2 and 3, which will allow us to study the corresponding cases of the second row.

\section{A. Scenario\#2: Case of No Cross-Layer Feedback}

In this part we will consider a receiver that does not support a cross-layer feedback. Then the application layer 
(i.e. the Kalman filter) does not have any knowledge of the quality of the communication link such as communication noise variance, $\sigma_{n}^{2}$. As a result, it assumes that those packets that are not dropped are noise-free. We will find the optimum way of dropping packets for such a receiver. To ease mathematical derivation of this scenario, we assume that the observation noise is negligible compared to the communication noise ${ }^{2}$. The estimation using a Kalman filter will then be as follows:

$$
\hat{x}[k+1]=\left\{\begin{array}{cc}
A \hat{x}[k] & \text { if } k^{t h} \text { packet is dropped } \\
A C^{-1} \hat{y}[k] & \text { if } k^{t h} \text { packet is kept }
\end{array}\right.
$$

This will result in the following recursion for the estimation error variance using Eq. 1:

$$
e[k+1]=A^{2} e[k]+Q-\frac{A^{2} e[k]-A^{2} C^{-2} \sigma_{n}^{2}(\Upsilon[k])}{S_{2}[k]},
$$

where $\sigma_{n}^{2}$ is the communication noise variance as defined in Section II and $S_{2}[k]=\left\{\begin{array}{cc}1 & \Upsilon[k] \geq \Upsilon_{T} \\ \infty & \text { else }\end{array}\right.$. Averaging Eq. 10 over $\Upsilon[k], \ldots, \Upsilon[0]$ will result in the following recursion for average estimation error variance:

$E(e[k+1])=A^{2} P_{L}\left(\Upsilon_{T}\right) E(e[k])+A^{2} C^{-2} P_{N}\left(\Upsilon_{T}\right)+Q$.

$P_{L}$ and $P_{N}$ represent average probability of packet loss and average communication noise variance that entered the estimation process respectively:

$$
P_{L}\left(\Upsilon_{T}\right)=E\left(P_{d r o p}\right)=\int_{0}^{\Upsilon_{T}} p d f(\Upsilon) d \Upsilon
$$

and

$$
P_{N}\left(\Upsilon_{T}\right)=\int_{\Upsilon_{T}}^{\infty} \sigma_{n}^{2}(\Upsilon) p d f(\Upsilon) d \Upsilon
$$

where $p d f$ represents probability density function.

1) Stability: It can be easily seen from Eq. 11 that the stability condition will be as follows:

$$
E\left(P_{\text {drop }, \text { scenario\#2 }}\right)<A^{-2},
$$

which means that the stability condition is independent of the shape of the communication noise profile. Furthermore, in order to maximize the stability range, all the packets should be kept in the receiver despite lack of a cross-layer feedback in this scenario. While keeping all the packets results in maximum stability range, it will not minimize the estimation error variance. Next we show how to design the receiver to minimize the estimation error variance.

2) Optimum Performance: The asymptotic average estimation error variance will be as follows as long as the stability condition of Eq. 14 holds:

$E(e[\infty])=\frac{A^{2} C^{-2} P_{N}\left(\Upsilon_{T}\right)+Q}{1-A^{2} P_{L}\left(\Upsilon_{T}\right)} \quad$ for $\quad P_{L}\left(\Upsilon_{T}\right)<A^{-2}$

\footnotetext{
${ }^{2}$ The analysis can be similarly carried out under the condition that the knowledge of observation noise variance, $R$, is not available in the estimator. Then $\sigma_{n}^{2}$ should be replaced by $\sigma_{n}^{2}+R$ throughout the analysis.
}

Intuitively, there should be an optimum $\Upsilon_{T}$ (optimum way of dropping packets) that will minimize the asymptotic average estimation error variance for this case. If $\Upsilon_{T}$ is too low, the receiver will keep most of the packets but the estimation will be too noisy. On the other hand, if $\Upsilon_{T}$ is too high, the receiver will be strict about the quality of the packets that it will keep. This reduces the amount of communication noise that enters the estimation process but will result in high packet loss rate and therefore information loss rate. Then the optimum $\Upsilon_{T}$ will be the one that provides a balance between information loss and communication noise.

Theorem 1 (Balance of Information Loss \& Communication Noise): Consider a receiver that does not support a cross-layer feedback. Let $\Upsilon_{T \text {, opt }}$ represent the optimum way of dropping packets which will minimize the asymptotic average estimation error variance of this receiver:

$$
\Upsilon_{T, o p t}=\operatorname{argmin} E\left(e\left[\infty, \Upsilon_{T}\right]\right)
$$

Then $\Upsilon_{T, \text { opt }}$ will be as follows:

$$
\Upsilon_{T, o p t}=\left\{\begin{array}{cc}
\Upsilon_{T}^{*} & \Upsilon_{T}^{*} \geq 0 \\
0 & \text { else }
\end{array}\right.
$$

where $\Upsilon_{T}^{*}$ is the unique solution to the following equation:

$$
\underbrace{P_{L}\left(\Upsilon_{T}^{*}\right)}_{\text {information loss }}+\underbrace{P_{N, \text { norm }}\left(\Upsilon_{T}^{*}\right)}_{\text {communication noise }}+\frac{C^{2} Q}{A^{2} \sigma_{n}^{2}\left(\Upsilon=\Upsilon_{T}^{*}\right)}=A^{-2},
$$

where $P_{N, \text { norm }}$ refers to the normalized average communication noise variance: $P_{N, \text { norm }}\left(\Upsilon_{T}^{*}\right)=\frac{P_{N}\left(\Upsilon_{T}^{*}\right)}{\sigma_{n}^{2}\left(\Upsilon=\Upsilon_{T}^{*}\right)}$.

Proof: Let $\Upsilon_{T}^{*}$ represent any solution to Eq. 18. By differentiating Eq. 15 with respect to $\Upsilon_{T}$, it can be easily verified that $\frac{\partial E\left(e\left[\infty, \Upsilon_{T}\right]\right)}{\partial \Upsilon_{T}}$ is only zero at $\Upsilon_{T}^{*}$. Next we show that Eq. 18 has a unique solution. Assume that Eq. 18 has two solutions: $\Upsilon_{T, 1}^{*}$ and $\Upsilon_{T, 2}^{*}>\Upsilon_{T, 1}^{*}$. Since $\sigma_{n}^{2}$ is a decreasing function of $\Upsilon_{T}$, the following can be easily verified:

$$
\begin{gathered}
P_{L}\left(\Upsilon_{T, 1}^{*}\right)+P_{N, \text { norm }}\left(\Upsilon_{T, 1}^{*}\right)+\frac{C^{2} Q}{A^{2} \sigma_{n}^{2}\left(\Upsilon=\Upsilon_{T, 1}^{*}\right)} \\
-P_{L}\left(\Upsilon_{T, 2}^{*}\right)-P_{N, n o r m}\left(\Upsilon_{T, 2}^{*}\right)-\frac{C^{2} Q}{A^{2} \sigma_{n}^{2}\left(\Upsilon=\Upsilon_{T, 2}^{*}\right)}<0
\end{gathered}
$$

Therefore, $\Upsilon_{T, 1}^{*}=\Upsilon_{T, 2}^{*}$. Let $\Upsilon_{T}^{c}$ be the critical stability Threshold: $1-A^{2} P_{L}\left(\Upsilon_{T}^{c}\right)=0$. Consider those cases where there exists a positive solution to Eq. 18. Then using the fact that

$$
\lim _{\Upsilon_{T} \rightarrow \Upsilon_{T}^{c}} E\left(e\left[\infty, \Upsilon_{T}\right]\right) \rightarrow \infty
$$

shows that $\Upsilon_{T}^{*}$ corresponds to the unique minimum of $E\left(e\left[\infty, \Upsilon_{T}\right]\right)$. Therefore, $\Upsilon_{T, o p t}=\Upsilon_{T}^{*}$. If process noise is the dominant noise, compared to the communication noise, there may be no positive solution to Eq. 18. It can be easily seen that, in such cases, $E\left(e\left[\infty, \Upsilon_{T}\right]\right)$ will be an increasing function for $\Upsilon_{T} \geq 0$, resulting in $\Upsilon_{T, o p t}=0$.

Theorem 1 shows that, in the absence of a cross-layer feedback, the optimum way of dropping packets is the one 
that provides a balance between information loss $\left(P_{L}\right)$ and communication noise $\left(P_{N}\right)$. To see the impact of operating at the optimum $\Upsilon_{T}$, Fig. 2 shows $E(e[\infty])$ as a function of $\Upsilon_{T}$ and for different levels of average Signal to Noise Ratio, $\Upsilon_{a v e}$. For this example, Signal to Noise Ratio, $\Upsilon$, is taken to have an exponential distribution and the communication noise profile is taken as follows: $\sigma_{n}^{2}(\Upsilon)=\alpha+\delta \times \zeta(\sqrt{\Upsilon})$, where $\zeta(c)=\frac{1}{\sqrt{2 \pi}} \int_{c}^{\infty} e^{-t^{2} / 2} d t$ for an arbitrary $c$. This is the variance of the communication noise for a uniformly quantized, BPSK modulation system [9]. The following parameters are chosen for this example: $A=2, C=1$, $N_{b}=10$ and $\Delta=.0391$, where $N_{b}$ and $\Delta$ represent the number of transmitted bits per packet and the quantization step size respectively. This results in $\alpha=1.27 \times 10^{-4}$ and $\delta=533.3$ [9]. Operating at the optimum $\Upsilon_{T}$ will result in reaching the minimums of the estimation error curves. It can be seen that dropping packets properly can improve the performance considerably. As $\Upsilon_{T}$ increases, the estimation will approach the instability regions due to high information loss as predicted by Eq. 14 .

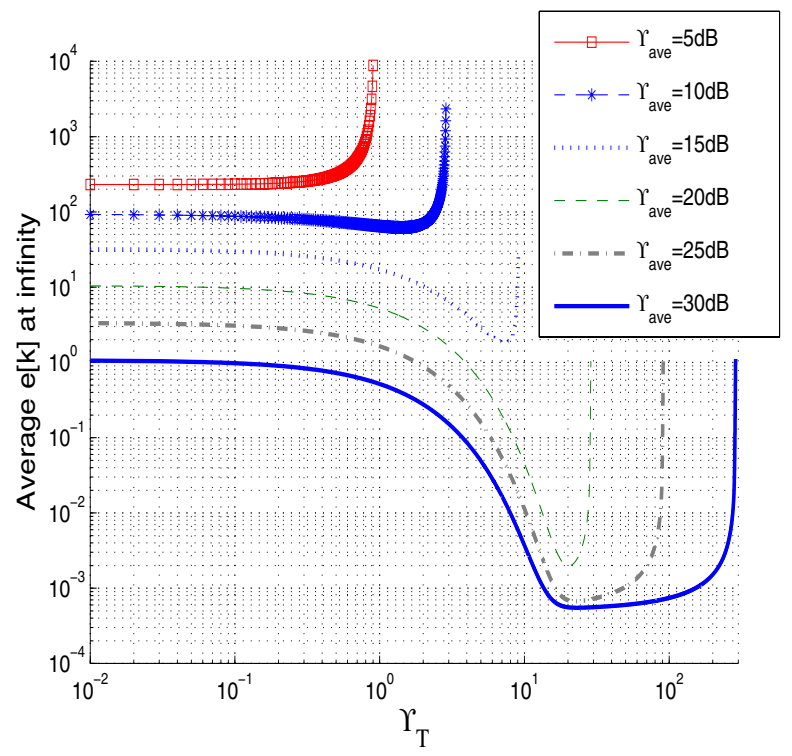

Fig. 2. Scenario\#2: Minimums of the curves indicating optimum way of dropping packets in the absence of a cross-layer feedback

\section{B. Scenario\#3: Impact of Cross-Layer Feedback}

Consider a receiver that can support a cross-layer feedback for those packets that are not dropped. This means that the Kalman filter will have the knowledge of the communication noise variance. We will have the following recursion for the estimation error variance:

$$
e[k+1]=A^{2} e[k]-\frac{A^{2} e^{2}[k] C^{2}}{C^{2} e[k]+\sigma_{z}^{2}(\Upsilon[k])}+Q,
$$

where

$$
\sigma_{z}^{2}(\Upsilon[k])=\left\{\begin{array}{cc}
\sigma_{n}^{2}(\Upsilon[k])+R & \Upsilon[k] \geq \Upsilon_{T} \\
\infty & \text { else }
\end{array}\right.
$$

\section{1) Stability}

The following two lemmas relate stability region of scenario\#3 to those of scenario\#1 and 2.

Lemma 1: The stability region of scenario\#1 includes that of scenario\#3:

$$
P_{L, c, \text { scenario } \# 1} \geq P_{L, c, \text { scenario } \# 3},
$$

where $P_{L, c}$ represents the maximum tolerable average probability of packet loss for stability.

Proof: Consider a special case of scenario\#1, where $R=0$. Let $g[k]$ and $e[k]$ represent the estimation error variances of scenario\#1 with $R=0$ and scenario\#3 respectively. Using Eq. 8 with $R=0$, we will have $E(g[k+1])=A^{2} P_{L} E(g[k])+Q$. From Eq. 21, it can be easily confirmed that $e[k+1] \geq A^{2} e[k]+Q-\frac{A^{2} e^{2}[k] C^{2}}{C^{2} e[k]+S_{1}[k]}$, where $S_{1}[k]$ is as defined in Eq. 8. This results in the following: $E(e[k+1]) \geq A^{2} P_{L} E(e[k])+Q$. Then,

$$
\text { if } E(e[k]) \geq E(g[k]) \quad \Rightarrow E(e[k+1]) \geq E(g[k+1])
$$

Therefore the stability region of scenario\# 1 includes that of scenario\#3.

Lemma 2: The stability region of scenario\#3 includes that of scenario\#2:

$$
P_{L, c, \text { scenario\#3 }} \geq P_{L, c, \text { scenario\#2. }} .
$$

Proof: Let $q[k]$ represent the estimation error variance of scenario\#2 for an $R \neq 0$. Then no knowledge of $R$ is available in the estimator for scenario\#2 (see footnote of Section IV, part A). Using Eq. 10, we will have:

$$
E(q[k+1])=A^{2} P_{L} E(q[k])+Q+A^{2} C^{-2} P_{N, R},
$$

where $P_{N, R}=P_{N}+\left(1-P_{L}\right) R$. Let $e[k]$ represent the estimation error variance of scenario\#3. We will have

$$
\begin{gathered}
E(e[k+1] \mid e[k])=\left(1-P_{L}\right) E\left(e[k+1] \mid e[k], \Upsilon[k]>\Upsilon_{T}\right) \\
+P_{L} E\left(e[k+1] \mid e[k], \Upsilon[k]<\Upsilon_{T}\right) .
\end{gathered}
$$

$e[k+1]$ is a concave function of $\sigma_{z}^{2}(\Upsilon[k])$ as indicated by Eq. 21. Therefore, using conditional Jensen's inequality, we will have,

$$
\begin{gathered}
E\left(e[k+1] \mid e[k], \Upsilon[k]>\Upsilon_{T}\right) \leq \\
A^{2} e[k]+Q-\frac{A^{2} e^{2}[k] C^{2}}{C^{2} e[k]+E\left(\sigma_{z}^{2}(\Upsilon[k]) \mid \Upsilon[k]>\Upsilon_{T}\right)} .
\end{gathered}
$$

Therefore,

$$
\begin{gathered}
E(e[k+1] \mid e[k]) \leq \\
A^{2} e[k]+Q+\frac{\left(P_{L}-1\right) A^{2} C^{2} e^{2}[k]}{C^{2} e[k]+E\left(\sigma_{z}^{2}(\Upsilon[k]) \mid \Upsilon[k]>\Upsilon_{T}\right)} .
\end{gathered}
$$

The third term on the right-hand side of Eq. 29 is a concave function of $e[k]$. Therefore by applying Jensen's inequality,

$$
\begin{gathered}
E(e[k+1]) \leq \\
A^{2} E(e[k])+Q-\frac{\left(-P_{L}\right) A^{2} C^{2} E^{2}(e[k])}{C^{2} E(e[k])+E\left(\sigma^{2}(\Upsilon[k]) \mid \Upsilon[k]>\Upsilon_{T}\right)}= \\
A^{2} P_{L} E(e[k])+Q+\frac{\left(1-P_{L}\right) A^{2} E(e[k]) E\left(\sigma_{z}^{2}(\Upsilon[k]) \mid \Upsilon[k]>\Upsilon_{T}\right)}{C^{2} E(e[k])+E\left(\sigma_{z}^{2}(\Upsilon[k]) \mid \Upsilon[k]>\Upsilon_{T}\right)} .
\end{gathered}
$$


Noting that $E\left(\sigma_{z}^{2}(\Upsilon[k]) \mid \Upsilon[k]>\Upsilon_{T}\right)=\frac{P_{N, R}}{1-P_{L}}$, it can be confirmed (using Eq. 26 and 30) that

$$
\text { if } E(e[k]) \leq E(q[k]) \quad \Rightarrow \quad E(e[k+1]) \leq E(q[k+1])
$$

Therefore the stability region of scenario\#3 includes that of scenario\#2.

Theorem 2: The stability condition will be as follows for a receiver that is equipped with a cross-layer feedback:

$$
E\left(P_{\text {drop }, \text { scenario\# } 3}\right)<A^{-2}
$$

Proof: Lemma 1 and 2 showed that $P_{L, c, \text { scenario\#2 }} \leq$ $P_{L, c, \text { scenario\# } 3} \leq P_{L, c, \text { scenario\# } 1 \text {. Noting that scenario\#1 }}$ and 2 have the same stability region proves Theorem 2 .

\section{2) Optimum Performance}

Theorem 3: Keeping all the packets, i.e. $\Upsilon_{T}=0$, will minimize the average estimation error variance for a receiver that is equipped with a cross-layer feedback.

Proof: Let $e_{1}$ and $e_{2}$ represent estimation error variances of two estimators using $\Upsilon_{T 1}$ and $\Upsilon_{T 2}$, where $\Upsilon_{T 2}>\Upsilon_{T 1}$. Then for any received Signal to Noise Ratio, $\Upsilon$, we will have $\sigma_{z, 2}^{2}(\Upsilon) \geq \sigma_{z, 1}^{2}(\Upsilon)$, where $\sigma_{z, 1}^{2}$ and $\sigma_{z, 2}^{2}$ are as defined in Eq. 22 for these two estimators. Using Eq. 21, the following can be easily confirmed for any given $\Upsilon[0], \Upsilon[1], \ldots, \Upsilon[k]$ :

$$
\begin{gathered}
\text { if } e_{1}[k] \leq e_{2}[k] \Rightarrow \\
\frac{e_{1}[k] \sigma_{z, 1}^{2}(\Upsilon[k])}{C^{2} e_{1}[k]+\sigma_{z, 1}^{2}(\Upsilon[k])} \leq \frac{e_{2}[k] \sigma_{z, 2}^{2}(\Upsilon[k])}{C^{2} e_{2}[k]+\sigma_{z, 2}^{2}(\Upsilon[k])} \Rightarrow \\
e_{1}[k+1] \leq e_{2}[k+1] .
\end{gathered}
$$

This shows that using a lower Threshold will result in a lower estimation error variance. Therefore, keeping all the packets, i.e. $\Upsilon_{T}=0$, will minimize the estimation error variance (and its average over the distribution of $\Upsilon$ ).

We can see that keeping all the packets not only prevents instability but also minimizes the estimation error variance in the presence of a cross-layer feedback. To see the impact of a cross-layer feedback, Fig. 3 shows average estimation error variance after 300 time steps for the system parameters of Fig. 2 and for both scenario\#2 and 3. By comparing the corresponding curves for these cases, it can be seen that a cross-layer feedback can improve the performance considerably even when compared to operating at the optimum $\Upsilon_{T}$ of scenario\#2. Furthermore, it can be seen that keeping all the packets will result in minimum average estimation error variance for scenario\#3. Finally, the stability condition of scenario\#3 is confirmed to be the same as predicted by Theorem 2.

\section{SUMMARY}

In this paper we considered estimation over mobile communication channels using a Kalman filter. We showed that the communication protocols suitable for other alreadyexisting applications like data networks may not be entirely applicable for estimation and control of a rapidly changing dynamical system. We provided receiver design principles for such delay-sensitive applications. More specifically we

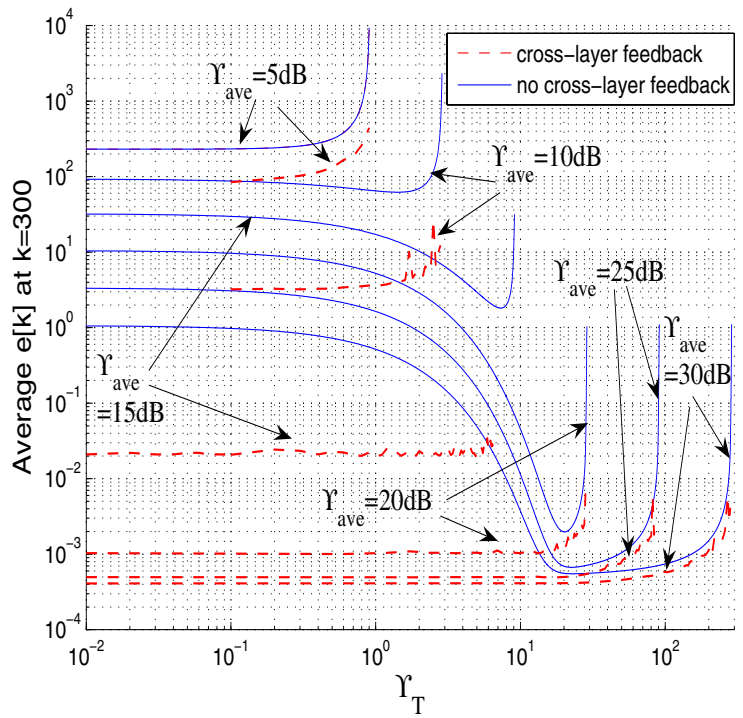

Fig. 3. Effect of cross-layer feedback: compare scenario\#2 and 3

derived stability conditions and investigated the performance of different receiver designs. We showed that stability condition is only a function of packet loss and is independent of the shape of the communication noise profile or availability of a cross-layer feedback. Therefore, in order to maximize the stability range, the receiver should keep all the packets independent of the quality of the link or availability of a cross-layer feedback. In the presence of a cross-layer feedback, this design will also minimize the estimation error variance. However, in the absence of such a feedback, we proved that packet drop should be designed to balance information loss and communication noise in order to optimize the performance.

\section{REFERENCES}

[1] C. Chong and S. Kumar, "Sensor networks: evolution, opportunities and challenges," Proceedings of the IEEE, vol. 91, issue 8, Aug. 2003, pages:1247-1256

[2] M. Micheli and M. I. Jordan, "Random sampling of a continuous-time dynamical systems," Proceedings of 15th International Symposium on the Mathematical Theory of Networks and Systems (MTNS), University of Notre Dame, South Bend, Indiana, 2002

[3] B.Sinopoli, L. Schenato, M. Franceschetti, K. Poolla, M. Jordan, S. Sastry, "Kalman filtering with intermittent observations," Proceedings of the 42nd IEEE Conference on Decision and Control, Dec. 9-12, 2003, Volume: 1, Pages:701 - 708

[4] X. Liu and A. J. Goldsmith, "Kalman filtering with partial observation losses," 43rd IEEE Conference on Decision and Control, 2004

[5] Y. Mostofi and R. Murray, "On dropping noisy packets in Kalman filtering over a wireless fading channel," Proceedings of the 24th American Control Conference (ACC), June 2005, Portland, Oregon.

[6] Theodore S. Rappaport, Wireless Communications, Principles and Practice. Prentice-Hall, July 1999

[7] W. Jakes, Microwave Mobile Communications. IEEE Press, 1974

[8] T. Kailath, A. H. Sayed, B. Hassibi, Linear Estimation. Prentice Hall information and system sciences series

[9] Y. Mostofi and R. Murray, "Effect of time-varying fading channels on the control performance of a mobile sensor node," Proceedings of IEEE 1st International Conference on Sensor and Adhoc Communications and Networks (SECON), Oct. 2004, Santa Clara, California. 